\title{
Brasil en el periodo azucarero
}

Rodrigo Espino, Raúl Martínez

$\mathbf{E}_{1}$ hecho histórico que marcó el inicio de la expansión portuguesa tuvo lugar en 1415: fue la toma de Ceuta. De esta forma, el peligro musulmán fue reducido a su mínima expresión y puso a los portugueses a las puertas del camino hacia el comercio de las especias. A partir de entonces, los portugueses avanzarían, paso a paso, a lo largo del siglo $\mathrm{XV}$, hasta llegar a la India. En las primeras etapas de la expansión lusitana, dos fueron sus aspectos importantes: la aportación de capital holandés y la formación de una especie de escuela en Sagres, la cual se abocó al estudio e investigación del comercio y de la exploración marítima. ${ }^{1}$

En 1441, luego de que Gil Eannes dobló el cabo Bojador, en África, una nave portuguesa logró traer oro en polvo y algunos esclavos negros. Con ello se inició el comercio de esclavos. Siete años después, el príncipe Enrique ordenó la construcción de la primera factoría europea en la isla de Arguim. El valor comercial de las exploraciones era ya evidente, al tiempo que cobraba importancia el aspecto religioso. Se estableció la costumbre de pedir permiso al Papa para la evangelización de los negros. "Esta costumbre de recurrir al Papa para la confirmación de todos los descubrimientos de ultramar fue un rasgo importante de la diplomacia de la época, y produjo más tarde embarazosas complicaciones, cuando España y otras naciones se lanzaron al campo de las exploraciones maritimas." 2 El papado, como máxima autoridad cristiana, adquirió presencia política internacional y se convirtió en legitimador de la estancia de los portugueses -y españoles más tarde- en tierras extraeuropeas.

Luego de la guerra contra España (1475) los portugueses, aunque la perdieron, gracias al tratado de Alcacovas (1479) lograron confirmar su monopolio sobre el comercio, colonización y exploración de las costas de África y la posesión de las islas del Atlántico -excepto las Canarias que siguieron en manos de los españoles.

Con la construcción del segundo fuerte en Elmina (1487), la circulación de mercancías africanas adquirió relevancia y aportó grandes utilidades al comercio portugués. En 1487 Bartolomé Díaz logró pasar el cabo de las Tormentas al que Juan II denominó de la Buena Esperanza. Con esto los portugueses avanzaron en su proyecto de llegar a la India, el cual culminan con el arribo de Vasco de Gama a Malindi y Calcuta en 1497, de donde regresó con pimienta y canela. Aun cuando los hindúes preferían comerciar con los musulmanes, a principios del siglo XVI los portugueses lograron dominar las principales rutas maritimas del Lejano Oriente hacia Europa.

En cuanto a la región marítima occidental, por el tratado de Tordesillas (1494) España y Portugal se dividen sus respectivas zonas de influencia. Dicho tratado confirma la presencia de los portugueses en Sudamérica. Posteriormente, las bulas papales de 1506 y 1514 dieron a los lusitanos el derecho de explorar y colonizar las nuevas tierras

'H. J. Parry, Europa y' la expansión del mundo (15/5-1715), trad. María Teresa F., México, Fondo de Cultura Económica, 1958, p. 33.

2 Ibidem, p. 36. 
que descubriesen, con lo que aseguraron su estancia en el continente americano ante los distintos monarcas europeos.

A raíz de la expedición encabezada por Pedro Álvarez Cabral, a principios de 1500 , los portugueses llegaron a la costa de lo que hoy conocemos como Brasil. El viaje de Cabral tuvo como objetivo conocer el potencial económicó de las costas sudamericanas. Por ese entonces, Portugal no estaba en condiciones de colonizar los territorios recién descubiertos, su interés consistía en encontrar un recurso aprovechable económicamente con un mínimo de inversión. Pronto se percataron de la existencia en las costas americanas dominadas por ellos, de un árbol de madera tintórea: el palo brasil. La Corona portuguesa, en un primer momento, dio concesión para la explotación del palo brasil a un solo individuo, Fernando de Noronha, a quien otorgó el título de capitán. Sin embargo, le fue retirada la concesión en 1504 ante las amenazas de Francia e Inglaterra, países que no reconocían la autoridad de Portugal sobre territorio americano, pues también se dedicaban a explotar el palo brasil. Tales circunstancias orillaron a Portugal a crear un sistema de guardacostas y posteriormente las llamadas factorias, que sirvieron como almacenes de palo brasil y, eventualmente, como puntos donde podían protegerse los barcos averiados por franceses e ingleses. Durante los 30 años que duró la explotación del palo brasil (1500-1530) fue utilizada fuerza de trabajo indigena. Ésta no sólo fue utilizada por los portugueses, sino también por sus adversarios, que incluso ocuparon ciertas áreas del territorio americano.

A partir de 1530, Portugal comenzó a plantearse la ocupación efectiva de su territorio americano. El rey Juan III otorgó a Martín Alfonso de Sousa poderes para la explotación de recursos, descubrimiento, conquista y colonización del territorio mencionado. De esa manera, se pueden distinguir dos fases en cuanto a la ocupación de Brasil: “... el reconocimiento y explotación de las costas, a base del comercio del palo brasil (y) el de la colonización propiamente dicha". ${ }^{3}$ En la primera se reproduce la experiencia de África e India, mostrándose ineficaz como en esas zonas por lo que en América, Portugal trató de colonizar su territorio, sobre todo ante las amenazas de Francia, Inglaterra y la propia España. Tal situación originó la creación de villas con el objetivo de asegurar la ocupación. Las primeras poblaciones fueron habitadas por lusitanos de escasos recursos que se situaron en el actual estado de São Paulo. Un ejemplo de estos asentamientos fue San Vicente, cuya fundación data de 1532.

Como una característica de los primeros años de ocupación "efectiva, tenemos la descentralización del poder, cuestión que se aprecia sólo en un periodo inicial. Portugal no tenía la menor intención de fragmentar el poder absoluto de la monarquía, trasladando a América instituciones de tipo feudal. En un primer momento las capitanías hereditarias se crean para ocupar el territorio brasileño y así promover su desarrollo económico. La Corona otorgó plenos poderes a sus capitanes generales, con el fin de que pudiesen controlar sus extensos territorios. Las capitanías proliferan y entre 1534-1536 se crearon 15 con 12 dueños. "Las capitanías brasileñas fueron dadas a hombres de importancia secundaria... Era una forma económica de retribuir servicios que no merecían premios de mayor valía." ${ }_{4}^{4}$ Poste-

\footnotetext{
${ }^{3}$ Eulalia María Lahmeyer Lobo, Administrafao colonial luso-espanhola nas Americas, Río de Janeiro, Editora Companhia Brasileira de Artes Gráficas, 1952, p. 117.

${ }^{4}$ Ibidem, p. 131
} 
riormente, la Corona trató de subordinar tanto como fuese posible a los capitanes. Asimismo, para evitar que se fragmentara su poder en la colonia, creó cuatro capitanías reales. La finalidad era estar presente ante sus capitanes generales y controlar el poder que se les había concedido garantizando, además, la soberanía real sobre la colonia. ${ }^{5}$

En 1538, el rey Juan III envió a Thomé de Souza como gobernador general del Brasil; fundó San Salvador, erigiéndola como capital de la colonia lusitana (1549) y procuró que los privilegios de las capitanías caducaran en beneficio de su gobierno, el cual dependía directamente de la Corona. Para 1553, Duarte Da Costa, sustituto de Thomé de Souza, llevó los primeros misioneros con el fin de frenar la conducta de los grandes latifundistas, "...cuya autoridad de horca y cuchillo no admitía más regulaciones para su conducta privada y pública que las impuestas por su propio capricho..."6 En $1558 \mathrm{Mem}$ de $\mathrm{Sa}$, gobernador general, ejerció una verdadera autoridad en Brasil. Sin embargo, la falta de recursos de la Corona impidió una efectiva centralización administrativa, favoreciendo así la independencia municipal, manteniéndose el poder de las capitanías. ${ }^{7}$ De esa forma, fue en las propiedades rurales donde se concentró la vida de la colonización, pues los núcleos urbanos incipientes dependían directamente de ellas, ya que la metrópoli se encontraba muy lejos para abastecerlos.

La donación de las capitanías por parte del rey respondió a un patrón establecido; por tanto, todos los capitanes tenían los mismos derechos, poderes y obligaciones. Entre los principales puntos de la donataria real tenemos: la donación de un $20 \%$ de las tierras de la capitanía; explotación de salinas, aguas y productos que eventualmente fuesen encontrados; $15 \%$ de las ganancias redituadas por el palo brasil; permiso para esclavizar indios y aun para exportarlos. Lo anterior en cuanto a lo económico. Por lo que respecta a lo jurídico, se daba al capitán jurisdicción legal y comercial; poder para la fundación de villas; se derogaron algunas obligaciones fiscales del capitán y de los súbditos, se estableció la obligación de servir al gobierno en caso de guerra, etc. En los puntos anteriores podemos observar el interés de la Corona en aprovechar al máximo los recursos económicos del territorio colonizado. ${ }^{8}$ Por otra parte, el donatario tenía que otorgar tierra a quien se lo solicitara - previo cumplimiento del diezmo-, concesión conocida como sesmaría. Sin embargo, en la práctica, para cultivar era necesario poseer esclavos, cuando menos así sucedía en el Nordeste. En estas condiciones, la sesmaría pasó a ser un privilegio discriminador. ${ }^{9}$ Todo ello se hacía con el objetivo de ocupar y hacer producir el territorio de la capitanía. Este sistema de ocupación ya había sido aplicado en Madeira y las Azores, por lo que se confiaba en obtener buenos resultados.

Las capitanías cubrieron todo el territorio brasileño confiriéndole una división política más o menos coherente, aun cuando algunos

\footnotetext{
${ }^{5}$ Ibidem, p. 141.

${ }^{6}$ Raúl Botelho Gosálvez, Proceso del subimperialismo brasileño, Argentina, EUDEBA, 1974, p. 22.

${ }^{7}$ Lahmeyer, op. cit., p. 230.

${ }^{8}$ Ivan Pedro De Martins, Iniroduçao a economia brasileira, Rio de Janeiro, Ed. Olympo, 1861, p. 45-46.

'Nelson Werneck, Sodré Formacao historica do Brasil, Sao Paulo, Ed. Brasilense, 1967, p. 71.
} 
beneficiarios no llegaron a usufructuar la donación otorgada por el rey. Un caso excepcional, en el sentido económico, lo constituyó el capitán Duarte Coelho, quien obtuvo la capitanía de Pernambuco, desde la cual se difundió, especialmente sobre el Nordeste, el cultivo y procesamiento de la caña de azúcar. A Coelho se le otorgaron 10 leguas dentro del territorio a partir de la costa y 60 leguas costeras, desde el río San Francisco hasta el río Santa Cruz. Cabe aclarar que nunca, por próspera que fuese la capitanía, se dejó anteponer los intereses personales por sobre los de la Corona.

Con la introducción del cultivo de la caña de ažúcar se terminó el ciclo económico del palo de brasil y dio inicio uno nuevo basado en aquel producto. Aunque los portugueses tenían la idea de encontrar oro, no despreciaron la oportunidad de explotar productos que les retribuyeran altas ganancias, al tiempo que con ello reforzaban la colonización en el Brasil. "El hecho de ser Portugal el mayor productor de azúcar, durante el siglo $\mathrm{xv}$, antes del descubrimiento de América, parece explicar por sí solo, que éste haya sido el género básico de la colonización." 10 A Portugal se le facilitó la explotación del azúcar en tierras brasileñas, gracias a la experiencia obtenida de sus plantaciones en las islas Azores. Aun cuando los portugueses probaron en Brasil varios productos agrícolas -algodón, tabaco, etc.-, fue la caña de azúcar el cultivo que rindió mejores resultados. En Pernambuco encontraron tierra negra (massape) apta para el cultivo de la caña. Aquí fue donde se desarrollaron las primeras plantaciones azucareras, basadas en la gran propiedad y en la mano de obra esclava, primero serían indígenas y más tarde negros de África. En un principio, la gran plantación fue autosuficiente, pues se daban parcelas a los esclavos para su subsistencia. Los animales de tiro y para consumo humano se producían en la propiedad, mientras la madera para la construcción de viviendas y para el refinamiento del azúcar se obtenía del desmonte. No obstante, al intensificarse la producción fue necesario que el esclavo se especializara con lo que aumentó el régimen de explotación, el cual tenía como característica una larga jornada de trabajo. "Tal sociedad esclavista, sentada sobre inmensos latifundios, no conocía más que dos clases: señores y esclavos. Pero andando el tiempo la desenfrenada actividad genésica de los 'señores de ingenio' en el norte y los fazendeiros en el sur, engendró multitud impresionante de mestizos..."11 No obstante, no se pudo conformar una clase media independiente, "porque casi todos sin excepción estaban al servicio de algún terrateniente o vivian a la sombra de su patriarcado, bien como medieros, artesanos o simples curiales encargados de labores administrativas". 12

Para finales del siglo xvi la producción y los productores de azúcar habían aumentado. Los capitanes y sesmarios con escasos recursos recurrieron al capital holandés, que resultó un apoyo inestimable para el incremento de la productividad. Por medio del financiamiento otorgado a los productores, los holandeses prácticamente se apropiaron de la tierra, transporte, refinación y comercialización del azúcar. Los holandeses hicieron posible, de esta manera, el crecimiento de la producción de azúcar en el Brasil, que pronto se perfiló como primer

10 Ibidem, p. 63

"Botelho, op. cit., p. 21.

': Ibidem, p. 26. 
productor azucarero. Aún más, Holanda controlaba casi todo el comercio que llegaba por mar a Europa, por lo cual esta nación estuvo en condiciones financieras para intervenir en el proceso de producción y distribución del azúcar lusobrasileña, situación que confirió experiencia a los holandeses, quienes a su tiempo crearon en las Antillas una próspera zona productora, retirándose de Brasil no sólo con experiencia sino con capital y tecnología para intensificar la producción azucarera. Esto ocurrió en el siglo xvir; mientras tanto el capital holandés jugaría un papel muy importante en el desarrollo de la industria azucarera brasileña. ${ }^{13}$

Organizadas las plantaciones azucareras, pronto constituyeron grandes unidades productoras, las cuales reunían en un mismo conjunto el trabajo productivo y un abultado número de individuos bajo la dirección del propietario o de un capataz.

Es la explotación en vasta escala la que, conjugando áreas extensas y numerosos trabajadores, se constituye como una única organización colectiva de trabajo y de la producción... Su elemento central es el ingenio, esto es, la fábrica donde se reúnen las instalaciones para el manipuleo de la caña y la preparación del azúcar. ${ }^{14}$

Concomitantemente con el crecimiento y organización de la plantación, surgieron zonas subsidiarias a la producción azucarera como la ganaderia, explotación forestal y zonas de producción de alimentos para los esclavos. En este último caso la plantación seguía valiéndose por sí sola; sin embargo, los alimentos crecieron al margen de la plantación, pero supeditados en su desarrollo a los designios que el mercado, clima, etc., depararan a la producción del azúcar.

Los portugueses no sólo llevaron azúcar al Brasil, sino también todo el bagage técnico necesario para su obtención. Fueron introducidos instrumentos de molienda, técnicas de refinación, embarque, etc. Para facilitar la salida de la producción azucarera fueron construidos los ingenios cerca de los puertos de exportación. En los ingenios (entendidos como las instalaciones donde se da el procesamiento final de la caña para la obtención del azúcar) encontramos cierto avance técnico tendiente a incrementar la productividad. No obstante, les fue imposible prescindir de la fuerza de trabajo del esclavo negro para mover la maquinaria. Se puede afirmar que en esta segunda etapa del proceso productivo azucarero, dejando de lado el transporte de la caña al ingenio, encontramos más nitidamente un desarrollo relativo de las fuerzas productivas. Como un ejemplo del avance de la economía azucarera, tenemos que los ingenios en Pernambuco, Itamaraca, Paraiba y Río Grande pasan de unos 70 o 75 que existían en 1600 a 166 para el año de $1629 .{ }^{15}$

Por otra parte, existió el arrendamiento de tierras a labradores que contaban con esclavos pero no con ingenios para el "refinamiento" del producto; no debemos olvidar que las mejores tierras estaban cerca del puerto de exportación y en manos de unos cuantos indivi-

${ }^{13}$ Celiso Furtado, Formación económica del Brasil, México, Fondo de Cultura Económica, 1974, p. 18-19.

${ }^{14}$ Caio Prado Junior, Historia económica del Brasil, Buenos Aires, ed. Futuro, 1960, p. $40-41$.

${ }^{15}$ Mauricio Goulart, A escravidao africana no Brasil, São Paulo, ed. Alfa-Omega, 1975 , p. 109. 
duos. La instalación de los ingenios implicaba una inversión elevada, por lo que a aquellos que los instalasen se les concedía la liberación de tributos, se les daba honores, títulos, etc. Durante este periodo, la esclavitud se convirtió en una condición de sobrevivencia para el colono europeo. La captura y el comercio de indígenas, actividad prohibida pero practicada ante el incremento en el precio y escasez de negros esclavos, se constituyó en una de las ocupaciones de los núcleos de población no dedicados a la producción azucarera. ${ }^{16}$

El trabajo esclavo que aparece en esta etapa histórica crea algunos problemas de conceptualización, pues está fuera de contexto, si no se toma en cuenta que aparece en la vinculación del sistema de plantación al mercado internacional por medio del mercantilismo portugués; es decir, el esclavismo se destinó a satisfacer las necesidades lucrativas de Portugal. Por tanto se debe tener en cuenta que si bien es cierto que el esclavismo es un elemento de otro modo de producción, en el siglo xvı los portugueses lo ven como un elemento que garantiza la producción con el mínimo gasto de mantenimiento. También es necesario considerar que se introduce el esclavismo debido a la poca mano de obra disponible en la metrópoli, la cual en caso de haber existido hubiera elevado considerablemente los gastos de producción, sobre todo por las condiciones históricas de su desarrollo. Esto sin contar los requerimientos objetivos que el portugués no cubría. Por otro lado, no hay que olvidar que Portugal en ese momento estaba regido por una conducta eminentemente mercantilista. En el contexto del mercantilismo, podemos entender cómo Brasil y el esclavo çontribuyen a la acumulación en los países europeos, de tal manera que aun sin oro en ese entonces (siglos XVI-XVII) aportan su cuota al capitalismo a partir de la transferencia del excedente que se realiza en la circulación, mediante la venta del azúcar, en primera instancia, de Portugal a Holanda y después la distribución que hacía esta última nación en el mercado europeo.

Una premisa del mercantilismo fue "el aprovechamiento del mayor número de fuentes con posibilidad lucrativa..."17 que no necesariamente debían ser metales preciosos. Los teóricos y estadistas del mercantilismo no confundían el oro y la plata con la riqueza de un país, trataron de retenerlos, es cierto, pero no por ellos mismos, sino como representantes de la riqueza y no porque fuera intrínseca en ellos, "por ello se preocuparon en conservar en sus tierras el dinero que amenazaba desaparecer con el comercio". ${ }^{18}$ El mercantilismo no es una doctrina absoluta, coherente y fija; encuentra variantes y una de ellas es la que Max Weber denominó mercantilismo monopolista de Estado, cuyos representantes más logrados fueron los países ibéricos. ${ }^{19}$ Por otra parte, el mercantilismo no fue una ciencia económica estructurada; el mercantilismo representa tendencias en las que cabe desde el liberalismo holandés hasta el mercantilismo monopolista de Estado. ${ }^{20}$

El mercantilismo de Portugal aparece nítidamente cuando este país

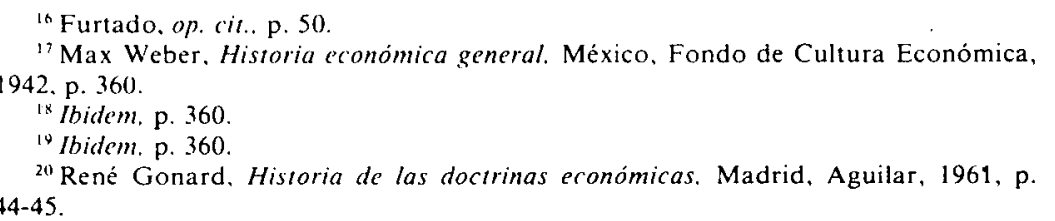


busca en Brasil desde las primeras exploraciones un producto altamente lucrativo hallando inicialmente en el palo brasil esas características. Posteriormente, en el azúcar se encuentra un nuevo y más lucrativo producto que tuvo amplia demanda en Europa en el siglo XVI. Por tal razón el objetivo de los productores azucareros fue conquistar el mercado europeo, donde su producto tenía gran valor y por ende era altamente lucrativa su comercialización. Todo ello en el contexto de la política mercantilista lusitana. ${ }^{21}$

No sólo debemos considerar la determinación económica sino también la política, que en Brasil como en Hispanoamérica marca importantes pautas de desarrollo; nos referimos a la relación metrópolicolonia. "El medio de acción del mercantilismo es ante todo la ordenanza o el edicto real" 22 , vínculo basado en la dominación colonial. El Estado toma en cuenta la necesidad de obtener recursos de sus colonias para lo cual no escatima la utilización del trabajo esclavo, la subordinación politica, la concesión de prebendas, incentivos fiscales, etc. Cabe mencionar que en Brasil no hubo feudalismo y aun cuando se encuentran instituciones con rasgos feudales, o el mismo esclavismo, se desarrollan aisladas, supeditadas al desarrollo mercantilista y capitalista comercial europeos; de ello depende su existencia. El mercantilismo tiene en España y Portugal su contraparte política, que es el absolutismo monárquico, y así como el mercantilismo es contrario a la doctrina económica medieval, la monarquía absoluta es contraria e incompatible con el feudalismo. ${ }^{23}$

En contraste con el desarrollo del Nordeste azucarero, las villas del sur de Brasil adoptaron otro tipo de características en su desenvolvimiento económico y social, pues sus condiciones no permitían la producción de azúcar. Tales villas giraban alrededor de la explotación del azúcar y de ello dependieron. Ún ejemplo es el caso de São Paulo, cuya población se dedicaba a la ganadería para abastecer de animales de tiro, cuero y carne a la zona azucarera. En esta región surgieron las bandeiras, integradas por paulistas con el fin de reconocer el territorio y explotar su posible potencial económico.

En São Paulo se implantó la pequeña propiedad y su población se formó de manera especial con hombres libres. No obstante las prohibiciones establecidas por la Corona, los paulistas establecieron relaciones mercantiles entre sí y aun con provincias españolas como la Banda Oriental y Buenos Aires. Como en São Paulo la prosperidad económica se reducía a los vínculos con la zona azucarera, sus habitantes trataron constantemente de ampliar los horizontes económicos explorando la selva. O bien cuando se dio la escasez de esclavos negros, los paulistas se dedicaron a la captura del indio, aun en las reducciones jesuitas de Paraguay, para venderlos como esclavos en la zona azucarera. Las bandeiras conocieron tres fases en su desarrollo: una exploratoria que dio cuenta del potencial de la zona; otra de población que se encargó de ocupar el territorio explorado con fines económicos, y una tercera variante, conocida como bandeiras predatorias que son las que expanden el territorio brasileño a costa de las reducciones jesuitas de Paraguay.

Muchos de los nuevos núcleos de población se dedicaron a la pro-

${ }^{2 !}$ Prado, op. cit., p. 36-37.

${ }^{22}$ Gonard, op. cit., p. 46 .

${ }^{23}$ Ibidem, p. 49. 
ducción de artículos de manutención -cultivos especializados como la mandioca, el maíz y el arroz. Se trató

de pequeñas unidades que se aproximan al tipo campesino europeo, en que es el propietario quien trabaja ayudado, cuando mucho, por un pequeño número de auxiliares, por lo general su: propia familia... La población indigena contribuye en gran parte a formar esta clase de pequeños productores autónomos. ${ }^{24}$

Por otra parte, la producción ganadera adquirió poco a poco relevancia para el consumo interno. Aunque actividad secundaria en la economía brasileña, la ganadería se extendió a lo largo del desierto del Nordeste, determinada por el incremento del consumo del litoral donde la producción azucarera y la colonización erán muy activas. La utilización de trabajo libre en las haciendas ganaderas implicaba relaciones de producción diferentes:

el hombre que trabaja en la hacienda ganadera durante cierto número de años (cuatro o cinco) tenía derecho a una participación (una cría de cada cuatro) en el rebaño en formación pudiendo así iniciar la cría de ganado por propia cuenta... Siendo la ganadería nordestina una actividad dependiente de la economía azucarera, en los primeros tiempos era la expansión de ésta la que determinaba el desarrollo de aquélla. ${ }^{25}$

La inversión en la actividad ganadera se reponía automáticamente, sin muchos gastos significativos. Además, las condiciones de trabajo y alimentación eran tales que propiciaban un fuerte crecimiento vegetativo de su propia fuerza de trabajo. Los ingresos monetarios se obtenían en esta actividad por medio de la venta de animales de tiro, cuero y carne.

El azúcar que se producía en Brasil se destinaba al mercado europeo, siendo ahí donde se realizaba la plusvalía absoluta extraída al trabajador esclavo. Así, el objetivo del productor -dueño de la plantación- se concretaba a obtener una ganancia sobre la inversión hecha en esclavos, semilla $\mathrm{e}$ instalaciones sin tomar en cuenta la tierra que les había sido donada. Para este periodo (principios del siglo xviI), la empresa azucarera era la actividad predominante en Brasil, al grado de que todo agricultor quería dedicarse a ese cultivo. La Corona decidió intervenir para regular la producción de alimentos, "obligando a los propietarios a plantar mandioca y otros alimentos; se gravan las donaciones de tierras con obligación de cultivar en ella artículos alimenticios desde el primer año de la concesión..."26 Se puede decir entonces que es la dinámica de la economía azucarera la que impuso determinaciones a las demás actividades económicas, a las que subordina y aprovecha las ventajas que tal situación podía ofrecer. Así, la cría de ganado quedó prohibida por la Corona en la faja del litoral, principal zona azucarera.

Las actividades azucarera y ganadera se relacionaban entre sí en el intercambio, llegando a conformar una unidad productiva vinculada

\footnotetext{
${ }^{24}$ Prado, op. cit., p. 45.

${ }^{25}$ Furtado, op. cit. p. 67.

20 Prado, op. cit. p. 45-46.
} 
en su dinámica al mercado europeo. Tal relación mercantil BrasilEuropa estaba mediada por la administración portuguesa, la cual se encontraba como reguladora de las actividades productivas en Brasil en un intento por poner orden en beneficio propio. De esa forma, la Corona trató de controlar tanto la unidad productiva azúcar-ganadería como la incipiente economia de carácter urbano, cuyo desarrollo se generó al amparo de la producción azucarera de exportación.

Los núcleos urbanos en crecimiento albergaron a hombres libres, especialmente europeos, quienes constituyeron su propia justicia, funciones de gobierno y de policía. Actividades como la herrería, carpinteria, textil, etc., estaban en manos de productores independientes, representados en la Cámara, la cual ejercía el poder legislativo (se encontraba en São Paulo), y fungía a la vez como garante de la autonomia del municipio. Se encargaba, asimismo, de reglamentar las manufacturas. La Corona subordinó al poder del gobernador general tanto las capitanías como las cámaras, lo cual suponía el monopolio real sobre las actividades económicas brasileñas. Los portugueses, en ese sentido, contaban con la experiencia monopolista ejercida sobre las mercancias orientales. Se puede decir que la administración portuguesa se convirtió en monopolista, pero sólo del intercambio mercantil Brasil-Europa y no de la producción directa brasileña, la cual siguió en manos de particulares. A pesar de ello, la Corona obtuvo beneficios económicos que utilizó, especialmente, para cubrir sus deudas.

La Corona portuguesa logró articular la forma de producción azucarera-pecuaria y la actividad generada en los núcleos urbanos al regular la comercialización. Tanto la producción de azúcar, como la de carne y cuero y la incipiente producción de los núcleos urbanos, se convirtieron en mercancías. Mercancías cuya dinámica de circulación se encontró regulada por la admínistración metropolitana, que a su vez se encontraba inmersa en las determinaciones económicas de la circulación mercantil europea. Tal cuestión mostraría, en lo general, el predominio de la forma de producción embrionariocapitalista europea en la formación económico-social brasileña.

Vista en su conjunto, la economía azucarera con base en el trabajo esclavo - como casi todos los factores de la producción son del empresario-, el ingreso monetario obtenido de la venta del producto pasa en su mayor parte a manos del dueño; dicho ingreso se expresaba en el valor de las exportaciones. El flujo del ingreso desde el exterior se reducía, en la economía azucarera, a simples operaciones contables, reales o virtuales. Esto ha inducido a mucha gente a creer que esa era una economía de tipo feudal, sin embargo,

el feudalismo es un fenómeno de regresión que traduce el atrofiamiento de una estructura económica. Este atrofiamiento resulta del aislamiento impuesto a una economía, aislamiento que engendra gran disminución de la productividad por la imposibilidad en que se encuentra el sistema de sacar partido de la especialización y de la división del trabajo que le permite el nivel de la técnica ya alcanzada ... [la unidad esclavista] a la inversa de la unidad feudal, ella vive totalmente de cara al mercado externo. La supuesta similitud deriva de la existencia de pagos in natura en una y otra. Pero todavía aquí existe una total equivocación, pues en la unidad esclavista los pagos a factores son todos de naturaleza monetaria, debiéndose 
tener en cuenta que el pago al esclavo sólo es aquel que se hace en el acto de la compra de éste. ${ }^{27}$

Además, las estructuras de dominación política y de apropiación económica de la sociedad esclavista brasileña estaban determinadas, en última instancia, por las exigencias de la producción de urła plusvalía. Ésta era producida por el trabajo esclavo, cuya enajenación,

.. .resultaba en su subordinación física y moral al señor (blanco) en su actividad productiva, en el producto de su trabajo y en sus actividades religiosas, lúdicas y otras. En esas condiciones, las estructuras de dominación eran, al mismo tiempo y necesariamente, altamente represivas y universales, estando presentes en todas las esferas prácticas e ideológicas de la vida del esclavo (negro, mulato, indio, mestizo). Así, la formación social esclavista era una sociedad bastante articulada internamente... ${ }^{28}$

Durante la época más próspera del ciclo del azúcar, en el Nordeste los ingenios en el sentido amplio del término están en manos de propietarios privados, pero supeditados al capital holandés que es prácticamente el đueño de la producción de caña de azúcar y del azúcar propiamente dicha. Al mismo tiempo Holanda controlaba la distribución en el mercado europeo. Sin embargo, encontramos que el poder dominante no viene del capital sino del polo político de poder: Portugal. El trabajo esclavo estaba caracterizado por su inserción al mercantilismo, el trabajador es propiedad privada junto con el resto de los instrumentos de trabajo, entre los cuales estaba considerado. Una característica de este trabajo es que da mayor valor al producto, pero tal valor se da únicamente cuando el trabajo del esclavo entra en el mercado europeo. El trabajo esclavo fue la base del lucrativo negocio de la producción de azúcar.

Las formas de producción adoptadas en las plantaciones azucareras - forma de producción dominante en Brasil- fueron de tipo esclavista, pero la utilización de maquinaria en los ingenios confiere a esa forma de producción un carácter de desarrollo económico de "transición" hacia una economía embrionariocapitalista. Al ser ésta dominante, penetrará y subordinará las relaciones de producción manifestadas en las distintas actividades productivas brasileñas (azucarera, ganadera y la incipiente producción urbana). Estas actividades, articuladas entre sí mediante la circulación mercantil, constituyeron una unidad productiva subordinada, y por tanto, determinada por el modo de producción capitalista incipiente europeo. Es pues durante el periodo que comprende el ciclo del azúcar - de mediados del siglo XVı a mediados del siglo xvil- cuando el capitalismo penetra en la formación económico-social brasileña, penetración mediada por la administración real portuguesa.

${ }^{17}$ Furtado, op. cit., p. 58.

${ }^{28}$ Octavio Ianni, Esclavitud y capitalismo, México, ed. Siglo XXI, 1976, p. 25. 